\section{$\underset{\substack{\text { hommes } \\ \text { \& migrations }}}{ }$}

\section{Hommes \& migrations}

Revue française de référence sur les dynamiques

migratoires

$1284 \mid 2010$

Migrations et environnement

Sénégal

\title{
Sénégal. Les pêcheurs migrants : réfugiés climatiques et écologiques
}

\section{Pierre Failler et Thomas Binet}

\section{(2) OpenEdition \\ 1 Journals}

\section{Édition électronique}

URL : http://journals.openedition.org/hommesmigrations/1250

DOI : 10.4000/hommesmigrations. 1250

ISSN : 2262-3353

\section{Éditeur}

Musée national de l'histoire de l'immigration

\section{Édition imprimée}

Date de publication : 1 mars 2010

Pagination : 98-111

ISSN : $1142-852 X$

\section{Référence électronique}

Pierre Failler et Thomas Binet, « Sénégal. Les pêcheurs migrants : réfugiés climatiques et écologiques », Hommes \& migrations [En ligne], 1284 | 2010, mis en ligne le 29 mai 2013, consulté le 20 avril 2019. URL : http://journals.openedition.org/hommesmigrations/1250 ; DOI : 10.4000/ hommesmigrations. 1250 


\section{Sénégal \\ Les pêcheurs migrants : réfugiés climatiques et écologiques}

Par Pierre Failler, senior research fellow et Thomas Binet, research associate, Centre for the Economics and Management of Aquatic Resources (CEMARE), Université de Portsmouth (Grande-Bretagne)

Port de pêche du quartier de Guet N'dar à Saint Louis, Sénégal ๑ D.R.

Au Sénégal, près de 15000 pêcheurs migrants originaires de la Casamance, de la petite ou de la grande côte, affrètent chaque année

leurs pirogues vers des destinations toujours plus lointaines. La raréfaction des bancs de poissons force les pêcheurs sénégalais à étendre leurs zones de pêche, parfois en conflit avec d'autres populations côtières. Or la gestion des ressources halieutiques de l'Afrique de l'Ouest est à son tour victime de cet éparpillement. Sans un cadre institutionnel adapté, il demeure difficile d'encadrer une pêche migrante dont l'avenir dépend de la préservation d'un écosystème menacé. 
Cet article a pour objet de renseigner l'évolution des processus migratoires le long des côtes ouest-africaines au cours des trois dernières décennies ${ }^{(1)}$. Les pêcheurs ouestafricains suivent les bancs de poissons au-delà de leurs rivages depuis qu'ils ont maîtrisé la navigation à la rame et à la voile. Ils ont, tour à tour, accompagné les bancs de poissons qui migraient, les capturant chemin faisant, et investi les zones de concentration de la faune aquatique. Les progrès technologiques aidant, les pêcheurs les plus aguerris, ghanéens et sénégalais surtout, se sont aventurés de plus en plus loin à partir des années cinquante, tout en continuant d'opérer depuis leur village natal. L'exode agricole massif lors des sécheresses récurrentes des années soixante-dix a déversé son lot de paysans dans l'un des seuls secteurs économiques en plein essor en Afrique de l'Ouest et pour lequel la qualification se limitait à savoir se servir de ses deux bras afin de haler au plus vite un filet ou un casier. C'est ainsi que la population des gens de mer ouest-africains s'est multipliée par trois ou quatre en moins d'une décennie et alimente ainsi les flux de pêcheurs migrants. L'essor des marchés d'exportation, dans la foulée de la convention de Yaoundé en 1963 et surtout celle de Lomé $^{(2)}$ en 1975, conforte les processus migratoires dans la recherche de poissons de haute valeur commerciale. La capture des espèces de faible valeur marchande est peu à peu cédée aux pêcheurs ne disposant que de pirogues rudimentaires et destinées à l'approvisionnement des marchés domestiques.

La mise en place progressive, à la fin des années soixante-dix, de zones économiques exclusives (ZEE), à la suite de la troisième conférence de l'Organisation des Nations unies sur le droit de la mer réunie à New York en décembre $1973^{(3)}$, avait contraint la liberté de migration des pêcheurs ouest-africains. Aux arrangements passés entre les représentants des pêcheurs et les chefs des villages côtiers aux abords desquels se déroulaient les activités de pêche se substituent des achats de licence de pêche au pays hôte. Ce changement radical ne condamne toutefois pas la pêche migrante ni n'avorte son explosion récente. La chute dramatique des ressources halieutiques dans les pays de tradition de pêche comme le Sénégal, du fait de captures au-delà de ce que les stocks de poissons sont en mesure de supporter sans péricliter, force de plus en plus de pêcheurs sédentaires à migrer vers des eaux plus poissonneuses. Ce que le faible modernisme des pêcheries de certains pays comme la Guinée-Bissau, le Sierra Leone ou encore le Liberia rend encore possible. Mais pour combien de temps encore ? La convoitise est telle aujourd'hui que l'abondance d'antan laisse place à la rareté des stocks de poissons. Les migrations de pêcheurs renvoient dès lors, de plus en plus, l'image d'un système qui court à la faillite et dont les déplacements ne sont qu'une fuite en avant. Les quelques tentatives récentes d'intervention publique, notamment la limitation du nombre de licences accordées aux pêcheurs étrangers, sont battues en brèche par les comportements clandestins des pêcheurs migrants aidés en cela par les 
trop faibles moyens de surveillance des pays. Aussi, tout en restant hors de portée des regards, la pêche migrante attire-t-elle aujourd'hui de plus en plus l'attention des décideurs publics de tous les pays de l'Afrique de l'Ouest.

\section{Quand les réfugiés climatiques des années soixante-dix deviennent pêcheurs}

Dès la fin du XIX siècle, les migrations de pêcheurs sénégalais s'étendent sur toute la sous-région. Elles gagnent en importance avec le développement de la petite économie marchande. Ainsi, au début du XX' siècle, les pays d'Afrique de l'Ouest passent progressivement d'un commerce triangulaire à une économie basée sur l'exportation de produits primaires (gomme arabique et produits du palmier, puis arachide, caoutchouc sauvage, café et cacao). En marge des grandes cultures de rente se développe une économie dite "informelle" par le biais

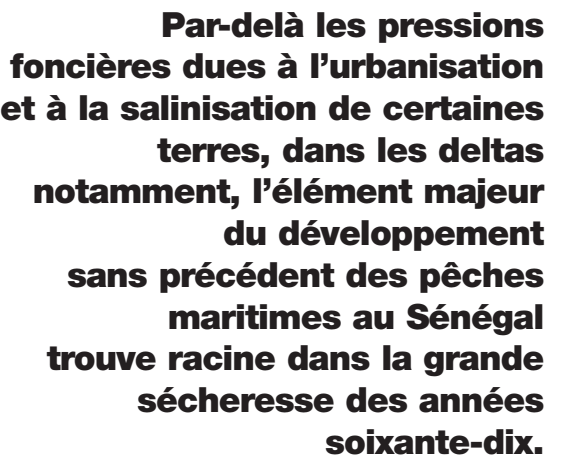
de petites exploitations agricoles écoulant leur production sur les marchés des centres urbains du littoral atlantique. Activité en développement, la pêche maritime approvisionne directement de tels marchés. On assiste alors progressivement au passage d'une pêche de subsistance fondée sur des échanges en nature, à une pêche commerciale (artisanale) orientée vers les marchés urbains.

Les nouveaux débouchés à l'exportation agissent comme des catalyseurs sur le développement des productions agricoles marchandes ainsi que sur la pêche migratrice à même de les satisfaire en débarquant des espèces de forte valeur commerciale. C'est ainsi que dans les années quarante à soixante, les pêcheries et unités de transformation européennes reposent de façon quasi exclusive sur l'approvisionnement par les pêcheurs piroguiers sénégalais ${ }^{(4)}$.

Par-delà les pressions foncières dues à l'urbanisation et à la salinisation de certaines terres, dans les deltas notamment, l'élément majeur du développement sans précédent des pêches maritimes au Sénégal trouve racine dans la grande sécheresse des années soixante-dix. La récurrence de ce phénomène a été telle qu'elle a provoqué un exode rural massif et a sonné, par la même occasion, la fin de l'agriculture de rente comme secteur moteur de l'économie sénégalaise. Les populations qui dépendaient de ces cultures ont dû trouver une nouvelle source de revenus et de nourriture. Le secteur des 
pêches maritimes alors en pleine expansion et doté d'une forte capacité d'accueil est peu à peu devenu un secteur "refuge" pour les laissés-pour-compte de l'agriculture. Ainsi, l'essor des pêches maritimes "résulte moins d'un choix délibéré que d'une reconversion à l'égard de l'agriculture ${ }^{(5)}$ ". On assiste dès les années soixante-dix à une migration environnementale forcée et massive des zones rurales vers le littoral. Dans les années quatre-vingt, la reconversion vers les pêches s'accentue encore avec la chute du prix des matières premières agricoles. La pêche artisanale voit ainsi sa main-d'ceuvre croître de 4 à 5 \% chaque année avec l'arrivée de jeunes ruraux de l'intérieur des terres.

\section{Modernisation et développement du secteur halieutique}

La forte capacité d'accueil du secteur est tout d'abord rendue possible par la démultiplication des pirogues et des engins de pêche, grâce à l'appui de divers bailleurs de fonds au développement des pêches au Sénégal. Ensuite, la démocratisation de l'usage des moteurs hors-bord sur les pirogues permettant des pêches lointaines provoque un engouement sans précédent pour la pêche migrante. Débutée dans les années cinquante sous l'effet de programmes nationaux de développement, la motorisation du parc piroguier sénégalais est quasi totale dans les années soixante-dix et participe alors largement de l'industrialisation du secteur ${ }^{(6)}$. Les pirogues peuvent ainsi gagner des zones de pêche plus au large et des régions côtières plus riches en ressources le temps d'une marée, avant de revenir débarquer au Sénégal où se trouvent les débouchés commerciaux. Cette motorisation s'accompagne notamment de l'utilisation de nouvelles techniques, comme les filets maillants dérivants pour la pêche des poissons démersaux et l'utilisation de glace à bord des pirogues, qui permettent d'accroître les rendements en restant sur les zones de pêche plusieurs jours durant. Enfin, et sur le plan commercial, la pêche migrante a fortement bénéficié de la convention de Lomé signée en 1975. Grâce au régime préférentiel mis en place, cette convention va offrir des potentialités, jusqu'alors inégalées, d'écouler les produits de la mer ouest-africains sur les marchés européens. Le Sénégal a ainsi augmenté considérablement sa fourniture de poissons (sous forme de matière première) aux usines de transformation européennes et de poissons de très haute valeur commerciale pour les places de frais comme Rungis à Paris, le marché aux poissons de la Porte de Tolède à Madrid et le Billingsgate à Londres.

En dépit des bouleversements des années soixante-dix et quatre-vingt, les pêcheurs migrants ont continué à suivre un cycle saisonnier, ponctué par les retours au village situé dans l'un des trois foyers principaux : Saint-Louis au nord du Sénégal, la 
presqu'île de Dakar et la petite côte proche du delta du Sine-Saloum. Les semailles, mais plus encore les récoltes, du riz notamment, sont l'occasion d'une pause à terre. Malgré ces retours, le mode de pensée agricole ${ }^{(7)}$ qui a longtemps prévalu dans la pêche s'efface peu à peu avec l'intensification progressive des migrations. Les pêcheurs ne sont plus des paysans-pêcheurs, exploitants d'un "terroir" de pêche, mais deviennent ce que Cormier-Salem appelle des pêcheurs du "parcours ${ }^{(8) "}$. Un parcours faisant intervenir un passage par les centres urbains littoraux, où la production est débarquée. À une échelle géographique plus large, si les aires de distribution de chaque foyer de migration sont variables, toutes s'étendent sur les zones maritimes des pays voisins : Mauritanie au nord et Gambie et Casamance au Sud. Les périples en Guinée-Bissau, en Guinée ou encore en Sierra Leone et au Liberia sont encore rares.

\section{L'élargissement de la zone de pêche face à la diminution des ressources}

La croissance des pêches sénégalaise est, dès la fin des années quatre-vingt, contrainte par la diminution de la taille et du nombre de bancs de poissons. Les principaux stocks de poissons exploités commercialement commencent à donner des signes de fatigue. Et ce qui prévaut à l'échelle de la ZEE nationale s'exprime à l'échelle de l'Afrique de l'Ouest tout entière du fait de la présence massive de flottes industrielles étrangères. Les captures des espèces d'intérêt commercial majeur subissent ainsi des diminutions allant de 20 à près de 40 \% entre 1996 et 2007. Les chiffres sont encore plus alarmants dans les eaux du Sénégal puisque les captures des espèces phares comme le thiof (mérou) accusent une chute de l'ordre de 80 \% entre 1990 et 2000, laissant penser que le seuil de rupture écologique a été atteint.

Devant la raréfaction des ressources, et afin de soutenir la production, les pêcheurs artisans sénégalais n'ont pas d'autres choix que d'aller plus loin afin d'exploiter de nouveaux stocks halieutiques. Cela est rendu possible par : des prix de vente très élevés pratiqués par les marchés d'exportation qui poussent les pêcheurs à investir davantage et à poursuivre le développement de leur activité de pêche ; des zones de pêche encore peu exploitées, c'est le cas de l'archipel des Bijagos en Guinée-Bissau, des îles Tristao et Alcatraz en Guinée où la pêche pratiquée par les populations autochtones n'en est encore qu'au stade de pêche de subsistance ; le caractère informel et souvent illégal des activités de pêche migrante, qui leur permet de contourner les conditions d'accès assorties à la création des ZEE à la fin des années soixante-dix et des accords de pêche entre pays de la sous-région pour la pêche artisanale. La faible capacité de contrôle maritime fait que le nombre d'embarcations en pêche dépasse celui autorisé par les protocoles d'accord. 


\section{Évolution des captures et niveaux d'exploitation} des principaux groupes d'espèces

\begin{tabular}{|l|c|c|c|}
\hline Espèces & Flottes* & $\begin{array}{c}\text { Évolution des } \\
\text { captures entre } \\
1997 \text { et 2006 }\end{array}$ & $\begin{array}{c}\text { Niveau } \\
\text { d'exploitation }\end{array}$ \\
\hline $\begin{array}{l}\text { Espèces démersales } \\
\text { (mérou, courbine, } \\
\text { sole, etc.) }\end{array}$ & $\begin{array}{c}\text { CV, Gui, Ma, Mo, } \\
\text { Sen, Sp }\end{array}$ & $-26 \%$ & $\begin{array}{c}\text { Modéré } \\
\text { à surexploitation } \\
\text { intense }\end{array}$ \\
\hline $\begin{array}{l}\text { Céphalopodes } \\
\text { (poulpe, octopus, } \\
\text { calamar) }\end{array}$ & Ma, Mo, Sen, Sp & $-31 \%$ & $\begin{array}{c}\text { Modéré } \\
\text { à surexploitation } \\
\text { intense }\end{array}$ \\
\hline $\begin{array}{l}\text { Espèces pélagiques } \\
\text { (sardines, sardinelles, } \\
\text { ethmalose, etc.) }\end{array}$ & $\begin{array}{c}\text { CV, Mo, PB, Sen, } \\
\text { Sp, Ukr }\end{array}$ & $-20 \%$ & $\begin{array}{c}\text { Pleinement exploité } \\
\text { à surexploitation } \\
\text { modérée }\end{array}$ \\
\hline $\begin{array}{l}\text { Crustacés (langouste, } \\
\text { crabe, crevette) }\end{array}$ & $\begin{array}{c}\mathrm{Fr} \text {, It, Mo, } \\
\text { Sen, Sp }\end{array}$ & $-38 \%$ & $\begin{array}{c}\text { Pleinement } \\
\text { exploité }\end{array}$ \\
\hline
\end{tabular}

${ }^{*} \mathrm{CV}=$ Cap-Vert, $\mathrm{Fr}=$ France, Gui $=$ Guinée, It $=$ Italie, $\mathrm{Ma}=$ Mauritanie, $\mathrm{Mo}=$ Maroc, Neth $=$ Pays-Bas,

Sen $=$ Sénégal, $\mathrm{Sp}=$ Espagne, Ukr $=$ Ukraine

Source : Failler et Gascuel, 2008

Se dessine alors une nouvelle forme de migration. Le caractère saisonnier s'estompe, tout comme les séjours au village pendant la saison des pluies. En mer toute l'année, les pêcheurs migrants s'établissent près des zones de pêche, dans des campements devenus permanents. Très jeunes pour la plupart, ils sont remplis d'un désir d'autoréalisation et d'accumulation de richesse, suffisamment motivant pour les tenir éloignés de leur village de façon quasi permanente. En d'autres termes, la migration leur permet de ne plus se plier à la coutume qui consiste à reverser leurs revenus à leur famille proche et élargie.

\section{Les nouvelles formes de pêche migrante}

Deux changements organisationnels de la pêche migrante sous-tendent l'implantation permanente des pêcheurs migrants dans les eaux des pays voisins ${ }^{(9)}$. Le premier s'articule autour du développement des campements de pêche totalement à l'écart des zones économiques et des routes de transport de marchandises, car situés dans les zones de mangroves ou sur les îles. Le second se réalise à partir d'un roulement de pirogues entre les lieux de pêche et les grands lieux de débarquement au Sénégal. 
104 Dossier | Migrations et environnement |

Carte des flux migratoires de la zone nord en 2008 
Dans le premier cas de figure, l'approvisionnement en carburant et en glace se structure non plus, comme auparavant, à partir des foyers de migration au Sénégal, mais depuis les débarcadères des pays hôtes. Certains postes d'approvisionnement sont même construits avec l'aide des coopérations internationales à des endroits proches des lieux de pêche comme à Canhabaque dans l'archipel des îles Bijagos. Dans la majorité des cas, la structuration des activités d'approvisionnement se fait depuis le campement même. Tout en se réapprovisionnant en vivres, essence et matériel de pêche, les pêcheurs peuvent y débarquer leurs captures. Disposant de plus d'unités de transformation du poisson (fumage, séchage) pour les espèces de faible valeur commerciale (petits pélagiques pour l'essentiel), les campements sont de véritables zones économiques reliées au pays d'origine par les va-et-vient continus des pirogues de transport acheminant à chaque trajet jusqu'à 30 tonnes de marchandises : poissons transformés dans un sens et essence, vivres et accastillage dans l'autre, sans oublier un flot de passagers à l'aller comme au retour.

La seconde forme de pêche migrante, moins visible car restant campée sur les zones de pêche sans jamais mettre pied à terre, n'en constitue pas moins la plus importante au plan économique. Elle concourt aujourd'hui à hauteur de 60 \% du volume de poisson exportée ${ }^{(10)}$ par le Sénégal à destination des pays de l'Union européenne (poisson de forte valeur commerciale pour l'essentiel). Organisée en marées d'une durée de 10 jours (qui correspond au temps de conservation du poisson sous glace sans détérioration), elle met à contribution deux pirogues armées de manière similaire : même capacité de stockage, même vitesse de déplacement et même nombre de matelots de manière à s'assurer d'une certaine uniformité des capacités de pêche. Pour la pêche organisée depuis les ports de la petite côte (M'bour et Joal) vers l'archipel des Bijagos, le

\section{L'explosion de la pêcherie de requin au Sénégal se heurte très vite aux limites physiologiques des sélaciens dont les cycles de reproduction sont très lents et limitent d'autant le renouvellement des stocks exploitables.} temps de la marée est partagé entre les deux jours nécessaires pour se rendre du port au lieu de pêche, les six jours à caler et haler les filets et les deux jours restants pour revenir au port de débarquement avec plus de 6 tonnes de thiof, carpes rouges, dorades grises ou roses dans les cales. Les deux pirogues qui opèrent ensemble se retrouvent donc tous les dix jours sur le lieu de pêche. Le passage de témoin entre la pirogue qui arrive et celle qui part se fait au-dessus du filet calé, qui ne quitte de la sorte jamais la zone de pêche! Si chaque pirogue est complètement autonome, la remontée de la pirogue pleine de poissons est conditionnée par l'arrivée de la seconde sur le lieu de pêche : une bonne coordination est ici un impératif de réussite. Les visites régulières 
aux ports sénégalais laissent penser que cette forme de pêche migrante prolonge quelque peu les formes organisationnelles des années soixante-dix. En réalité, il n'en est rien, car hormis les pêcheurs qui ont des liens familiaux dans les ports de Ziguinchor, Joal, M'bour, Soumbédioune, St-Louis, tous les autres continuent d'être déconnectés de leur village natal.

Tout comme dans les années soixante-dix, l'ouverture de nouveaux marchés permet de soutenir les migrations. Par exemple, la pêche du requin, déjà pratiquée de manière traditionnelle, s'intensifie dans les années quatre-vingt-dix sous l'effet de l'attraction exercée par le marché asiatique avec des prix atteignant 350 euros le kilo. Localement, ces filières sont intégrées verticalement ${ }^{(1)}$ : les mareyeurs (intermédiaires entre les pêcheurs et les exportateurs en gros) financent les campagnes de pêche et encouragent une reconversion des pêcheurs vers cette pêcherie. L'explosion de la pêcherie de requin au Sénégal se heurte très vite aux limites physiologiques des sélaciens dont les cycles de reproduction sont très lents et limitent d'autant le renouvellement des stocks exploitables. Aussi, la stratégie des pêcheurs sénégalais a-t-elle été à nouveau de se tourner vers les ZEE des pays voisins : gambienne, bissau-guinéenne, guinéenne et sierra-léonaise. La production d'ailerons pouvant être séchée et conservée à bord tout en ne nécessitant pas de débarquement régulier, les pêcheurs migrants sont extrêmement mobiles. 
Sur le plan spatial, les nouvelles formes de migration des pêcheurs sénégalais, émergeant à la fin des années quatre-vingt et qui se sont épanouies au cours de la décennie suivante, sont aujourd'hui omniprésentes dans toute la sous-région. Elles ont toujours pour racines les mêmes foyers, grâce au développement de leurs infrastructures portuaires, et ont dorénavant pour aire de répartition géographique: la Mauritanie depuis le foyer de Saint-Louis ; la Casamance et la Gambie depuis celui de la presqu'île de Dakar ; l'archipel des Bijagos, le sud de la Guinée-Bissau et le nord de la Guinée pour le foyer de la petite côte. Sur le plan économique, les estimations les plus récentes font état de près de 100000 tonnes de poissons capturés par les migrants sénégalais dans les ZEE des pays voisins (pour 400000 tonnes débarquées chaque année), soit un quart du total des captures du pays. C'est dire leur importance!

\section{Les migrations dans une impasse écologique et sociale}

Pendant longtemps les pêcheurs ont migré afin de suivre les déplacements d'espèces migratrices (petits pélagiques pour l'essentiel) ou de capturer des espèces qui se retrouvaient en abondance à un moment et un lieu donnés (lors de la période de frai, par exemple). L'adaptation des pêcheurs à la disponibilité des ressources a montré la forte capacité d'initiative de la pêche artisanale. Les migrations ont opéré un redéploiement de l'effort de pêche des flottilles d'une espèce à une autre, en l'orientant à chaque fois vers les stocks momentanément en plus forte abondance. En cela, elles favorisaient une bonne distribution spatiale et temporelle de l'effort de pêche et confirmaient le principe de la migration comme moyen de gestion flexible des pêcheries.

Ce principe est toutefois remis en cause depuis une décennie puisque les pêcheurs ont recours à la migration afin de pallier les insuffisances de poissons dans leurs zones de pêche traditionnelles. Migrer ne signifie plus alors mieux diriger l'effort de pêche d'une espèce à une autre au moment opportun, mais se déplacer faute de mieux. Dans le contexte actuel de raréfaction des ressources halieutiques et de déclin substantiel des principaux stocks de poissons tout au long de la côte ouest-africaine, la migration apparaît ainsi d'avantage comme un pis-aller qu'un arbitrage optimal entre différents choix de pêche.

La volonté politique actuelle de gérer les pêches artisanales, tant à l'échelle des pays qu'à celle de la sous-région dans son ensemble, amène à porter une attention nouvelle aux phénomènes migratoires. La majorité des captures réalisées par les 
pêcheurs artisanaux étrangers ne sont pas connues et donc non comptabilisées dans les statistiques nationales. Lorsque les débarquements se font dans le pays d'origine ou que les débarcadères sont trop éloignés pour être couverts par les enquêtes statistiques, aucune donnée quantitative n'est disponible dans le pays de capture. Lorsqu'ils sont comptabilisés, leur provenance n'est pas mentionnée : tout poisson débarqué est alors assimilé à un poisson capturé dans les eaux nationales. Les statistiques nationales sont dès lors faussées en attribuant à la ZEE nationale le poisson provenant de la ZEE voisine. Le pays qui bénéficie des débarquements de la pêche migratrice aura ainsi l'impression que ses stocks de poissons sont en meilleur état qu'ils ne le sont réellement; impression partagée par le pays où sont capturés les poissons du fait de l'absence de déclaration de captures. Difficile dans une telle situation d'émettre des avis scientifiques et de formuler des plans de gestion.

\section{La difficile cohabitation des pêcheurs migrants et autochtones}

Depuis la mise en place des ZEE nationales à la fin des années soixante-dix et au début des années quatre-vingt, l'accès des pêcheurs migrants aux eaux des pays tiers est régi par des accords de pêche entre pays. Ils portent pour l'essentiel sur un nombre d'embarcations autorisées à pêcher, mais nullement sur les espèces et les quantités permises. La faible capacité de contrôle maritime et la corruption font que le nombre d'embarcations en pêche dépasse celui autorisé par les protocoles d'accords. À ce phénomène se juxtapose un autre, sensiblement plus dommageable : celui de la pêche illégale dans les aires marines protégées. Que ce soit dans les réserves de Biosphère comme l'archipel des îles Bijagos, le Parc du Banc d'Arguin (à un degré moindre) ou encore dans les aires marines protégées en cours de création, comme celle de Tristao et Alcatraz, les activités de pêche des migrants sont en total désaccord avec l'esprit de protection de l'environnement marin ${ }^{(12)}$. Elles causent des dégâts écologiques, économiques et sociaux importants. L'absence totale de considération écologique de la part des pêcheurs migrants porte atteinte à la survie des stocks ciblés et de ceux dont les espèces sont capturées accidentellement. La problématique des migrations s'inscrit donc aujourd'hui dans le double jeu de la régulation de l'accès aux ressources halieutiques et celui de la conservation des écosystèmes marins au sein des aires marines protégées.

Les activités des pêcheurs migrants se situent par ailleurs chaque année davantage dans les zones traditionnellement fréquentées par les pêcheurs autochtones, ce qui entraîne une baisse de leurs captures, voire un retrait pur et simple de ces derniers de 
ces aires de pêche. Ainsi, et en dépit de la contribution de la pêche migrante au développement économique des communautés avec lesquelles ils cohabitent, en contribuant notamment à la création d'emploi, les échanges entre populations locales et pêcheurs étrangers laissent peu à peu la place à un rejet accru des allochtones, tant la fréquence et l'intensité des conflits entre pêcheurs autochtones et étrangers devient forte. Lorsque les instances locales ou les agences de contrôle des pêches sont mobilisées pour arbitrer les conflits, c'est souvent au détriment des pêcheurs migrants, dépourvus de quelques droits que ce soit, les mettant ainsi à la merci de sanctions abusives et de l'obligation de dessous-de-table. Même pour les migrants établis depuis plusieurs décennies dans les campements ou dans les villages, au moindre conflit, leur situation d'étranger est tout de suite pointée du doigt. Selon Dia, le comportement foncièrement non conforme aux normes sociales des communautés autochtones malgré les efforts d'intégration (mariages avec partenaires locaux, adoptions de coutumes, etc.) constitue le principal facteur d'explication. Il convient donc à la pêche migrante de légitimer sa présence dans les eaux des pays ouest-africains afin de pouvoir bénéficier de droits conséquents.

\section{Conclusion}

La sécheresse des années soixante-dix a provoqué un exode rural massif, venant gonfler les effectifs de la pêche ouest-africaine, et de la pêche migrante sénégalaise en particulier. Les pêcheurs migrants des régions de Saint-Louis, Dakar ou de la petite côte, réfugiés climatiques pour bon nombre d'entre eux, ont su avec profit tirer parti de l'abondance des poissons dans les eaux des pays voisins, aidés en cela par les programmes de développement initiés par les pouvoirs publics et les organisations internationales, ainsi que l'ouverture de marchés à l'exportation. Au cours des deux dernières décennies, les phénomènes migratoires se sont amplifiés, tant spatialement, temporellement que numériquement. Les zones de pêche se sont progressivement éloignées des lieux de résidence des pêcheurs. Au caractère saisonnier et épisodique des migrations se sont substituées une continuité et une présence permanente sur les zones de pêche. Face à l'effondrement des stocks de poissons au Sénégal, le nombre de pêcheurs affectés par les phénomènes migratoires est en progression constante. Les pêcheurs migrants sénégalais sont ainsi peu à peu devenus des réfugiés écologiques. On peut s'interroger dès lors sur ce qu'il adviendra si rien n'est fait dans les prochaines années pour cadrer et limiter ce phénomène : des migrations plus lointaines encore? Des pratiques plus intenses sur les zones de pêche? De nouvelles espèces ciblées? Davantage de pêche illégale dans les zones protégées ? Et pour les migrants, une vie à 
bord des pirogues? Une apatridie définitive ? Les pouvoirs publics commencent à prendre la mesure de l'ampleur du phénomène migratoire, longtemps tu, faute de chiffres. La coopération régionale, seule issue possible tant la distribution spatiale du phénomène dépasse les frontières d'un seul pays, semble s'organiser à partir du réseau des aires marines protégées de l'Afrique de l'Ouest (le RAMPAO): seule entité disposant aujourd'hui de fonds suffisant à même de juguler les intrusions permanentes des pêcheurs migrants dans les zones de protection.

La variabilité climatique, qui provoque, entre autres, une remontée vers le nord des circuits migratoires des principales espèces de poissons pélagiques ou encore l'apparition de nouvelles espèces démersales (poisson-chat en Mauritanie), va obliger les pêcheurs migrants à développer de nouvelles stratégies et globalement une nouvelle organisation. Cette adaptation aux changements, basée sur une forte capacité de résilience, leur conférera à nouveau le statut de réfugiés climatiques, délaissé dans les années quatre-vingt.

\section{Bibliographie}

- T. Binet, P. Failler et R. Bailleux, État des lieux et évolution récente des migrations de pêcheurs artisans en Afrique de l'Ouest, rapport final, Centre for the Economics and Management of Aquatic Resources, University of Portsmouth, rapport commandé par l'UICN, 2009 (à paraître).

- T. Binet, P. Failler, Rapport préliminaire, Quantification des phénomènes migratoires des pêcheurs artisans en Afrique de l'Ouest, rapport UICN, Dakar, Sénégal, 2010 (à paraître).

- J.-P. Chauveau, "Géographie historique des migrations de pêche dans la zone du Copace (fin XIX siècle - années quatre-vingt", in J. M. Haakonsen and M. C. Diaw, Migrations des pêcheurs en Afrique de l'Ouest, FAO, DANIDA, Norway, 1991.

- M.-C. Cormier-Salem, "Paysans-pêcheurs du terroir et marins-pêcheurs du parcours. Les géographes et l'espace aquatique", in L'Espace géographique, n 1, 1995, pp. 46-59.

- M.-C. Cormier-Salem, "Appropriation des ressources, enjeu foncier et espace halieutique sur le littoral ouest-africain", in J.-P. Chauveau, E. Jul-Larsen et C. Chaboud, Les pêches piroguières en Afrique de l'Ouest : pouvoirs, mobilités, marchés, Paris, CMI, IRD, Karthala, 2000.

- K. Dahou, et M. Deme, Accords de pêche UE-Sénégal et commerce international: respect des réglementations internationales, gestion durable des ressources et sécurité alimentaire, Dakar, Enda, 2002.

- A. D. Dia, "Réseaux et groupes d'appartenance chez les pêcheurs migrants: le cas des wolofs de N'Diago (Mauritanie), in Bulletin scientifique du centre national de recherches océanograpiques et des pêches n²8, 2001, pp. 55-68.

- P. Failler, G. van de Walle, M. Deme, A. Diop, D. Balbé, A. D. Dia, A. Bakalakiba, "Les aires protégées estuariennes, côtières et marines (APECM) en Afrique de l'Ouest : des réservoirs de ressources aquatiques en sursis", Revue Africaine des Affaires Maritimes et des Transports, Juillet 2009, n²1, 2009, pp. 43-50.

- P. Failler et Didier Gascuel, "Over-Exploitation in West Africa’s Richest zones”, in African Report, n¹2, 2008 , p. 28. 
- I. Odotei, "Migration des pêcheurs Fanti. Migrations des pêcheurs en Afrique de l'Ouest", J. M. Haakonsen and M. C. Diaw, Migrations des pêcheurs en Afrique de l'Ouest, FAO, DANIDA, Norway, 1991.

- R. Overa, "Institutions, mobility and resilience in the Fante migratory fisheries of West Africa. Population, Consumption and Environment Initiative (PCE)", Programme on Global Security and Sustainability, The MacArthur Foundation. Bergen, Norway, Chr. Michelsen Institute, 2000, p. 38.

- A. Sall, et P. Morand, "Pêche artisanale et émigration des jeunes africains par voie piroguière", in Politique Africaine, $\mathrm{n}^{\circ} 109,2008$

\section{Notes}

1. Cet article présente certains résultats d'un travail relatif aux migrations de pêcheurs artisans en Afrique de l'Ouest réalisé dans le cadre du projet de "Renforcement des capacités régionales de gestion de la pêche en Afrique de l'Ouest" (RECARGAO) de l'UICN et de la CSRP (Commission sous-régionale des pêches), coordonné par Renaud Bailleux. Ce projet est lui-même une partie du Programme régional de conservation de zone côtière et marine en Afrique de l'Ouest (PRCM). Les auteurs ont également bénéficié du soutien financier du programme européen de recherche internationale en coopération ECOST (Ecosystems, Societies, Consilience, Precautionary Principle: Development of an Assessment Method of The Societal Cost for Best Fishing Practices and Efficient Public Policies) pour l'approfondissement de certains éléments d'analyse. Le présent article ne reflète toutefois pas les positions de l'UICN et de la Commission européenne et n'anticipe pas sur leurs politiques futures dans ce domaine.

2. En favorisant l'exportation des matières premières des pays membres du groupe Afrique-Caraïbe-Pacifique (48 en 1975) à destination de l'Europe (exonération des droits de douane et absence de quotas).

3. Les travaux de cette conférence se sont achevés en 1982 par la signature à Montego Bay (Jamaïque) de la Convention des Nations unies sur le droit de la mer (CNUDM).

4. Lire à ce propos Anita Conti, Géants des mers chaudes, Éditions Payot, 2002, 244 pp.

5. J.-P. Chauveau, "Géographie historique des migrations de pêche dans la zone du COPACE (fin XIX siècle - années quatre-vingt)", in J. M. Haakonsen et M. C. Diaw, Migrations des pêcheurs en Afrique de l'Ouest, FAO, DANIDA, Norvège, 1991.

6. Le développement du secteur est alors largement soutenu par les pouvoirs publics et les organisations internationales qui y voient une étape transitoire vers l'industrialisation du secteur des pêches artisanales.

7. Activité rythmée par les saisons et structurée spécialement en terroir et à une échelle plus grande en territoire.

8. M.-C. Cormier-Salem, "Paysans-pêcheurs du terroir et marins-pêcheurs du parcours. Les géographes et l'espace aquatique", in L'Espace géographique, ${ }^{\circ} 1,1995$, p. 46-59 ;

M.-C. Cormier-Salem, "Appropriation des ressources, enjeu foncier et espace halieutique sur le littoral ouest-africain", in J.-P. Chauveau, E. Jul-Larsen and C. Chaboud, Les pêches piroguières en Afrique de l'Ouest : pouvoirs, mobilités, marchés, Paris, CMI, IRD, Karthala, 2000. Le terme de "parcours" est utilisé dans le même sens que celui de parcours des éleveurs pasteurs nomades. Il désigne : "Un espace de migration, fait ditinéraires et de réseaux sociaux et spatiaux, articulé par des routes autour de 'noeuds'stratégiques, contrôlé par une communauté qui en défend l'accès et les passages".

9. Une troisième forme de pêche migrante émerge depuis le milieu des années quatre-vingt-dix. Elle consiste à servir, avec l'aide d'une trentaine d'autres pirogues, d'unité de pêche à un navire industriel (baptisé pour l'occasion navire-collecteur) et à l'accompagner au fil de ses déplacements. Le peu de documentation rend toutefois difficile la description d'une telle pratique.

10. Environ 80000 tonnes. T. Binet et P. Failler, Rapport préliminaire, Quantification des phénomènes migratoires des pêcheurs artisans en Afrique de l'Ouest, rapport UICN, Dakar, Sénégal, 2010 (à paraître).

11. L'intégration verticale est une stratégie économique par laquelle un même acteur contrôle plusieurs niveaux de la filière pour s'assurer de son approvisionnement ou réduire ses coûts de production. Dans les pêcheries artisanales, des financeurs peuvent ainsi subventionner l'achat d'embarcations et en même temps offrir des débouchés aux pêcheurs ; c'est le cas de la filière des ailerons de requins pour le marché asiatique.

12. Tout comme le fumage de poisson à partir de la coupe de tonnes et de tonnes de bois de mangrove concourt à la fragilisation de la frange littorale. 